\title{
ANÁLISIS DE LA INFLUENCIA DEL ESPACIO PÚBLICO EN LA CALIDAD AMBIENTAL URBANA DE BOGOTÁ A TRAVÉS DE SIG
}

\author{
Leonardo Leguizamón Chaparro \\ Angie Viviana Torres Garzón \\ Ingeniería ambiental y sanitaria por la Universidad de la Salle. Vinculados al \\ semillero de investigación Sistemas de Información Geográfica para la Mode- \\ lación y Manejo Ambiental (SIGMMA).
}

\section{Resumen}

La población urbana en Colombia alcanzó en el año 2011 el 76\% de la población total nacional y se espera que llegue en menos de una década al 80\% según el Ministerio de Ambiente y Desarrollo Sostenible (MADS, 2012). Esta situación ha intensificado problemáticas ambientales en las áreas urbanas, manifestándose a través de cambios en el uso del suelo urbano, transformación y degradación del paisaje natural, y el deterioro del espacio público.

En el año 2008, el Ministerio de Ambiente estableció la Política de Gestión Ambiental Urbana y para dar cumplimiento de esta en sus objetivos consolidó la propuesta del Índice de Calidad Ambiental Urbana ICAU; el cual es una herramienta que posibilita tener un conocimiento más amplio del estado actual de las principales ciudades de Colombia en cuanto a calidad ambiental urbana, y de esta forma obtener una mejor planeación de las ciudades. Este índice ha sido implementado desde el año 2013 en las ciudades que cuentan con más de 500.000 habitantes: Bogotá, Medellín, Cali, Ibagué, Bucaramanga, Barranquilla, Cúcuta, Cartagena y Soledad.

La superficie verde urbana por habitante y el espacio público efectivo por habitante son dos de los indicadores directos incluidos en el ICAU que se manejan de forma independiente, motivo por el cual se propone hacer una unificación de dichos indicadores mediante al uso y análisis de datos de coberturas con el fin de sintetizar en un nuevo indicador cuyo contenido sea más representativo, pues es evidente que tanto las plazas y plazoletas públicas como las áreas verdes, entendidos como espacios urbanos contribuyen a la calidad de vida de los habitantes. De esta manera, se busca determinar de qué forma están distribuidas estas áreas dentro de la ciudad y qué particularidades se pueden identificar en cada sector para diagnosticar si efectivamente las zonas duras son un problema urbano o si las áreas verdes están más asociadas a la calidad de vida de los habitantes.

Por último, al ser un proyecto en desarrollo, no se cuenta todavía con los productos cartográficos que evidencien la incidencia de estos indicadores en la calidad ambiental urbana de la ciudad de Bogotá. 


\section{Introducción}

En Colombia, desde el año 2013 se ha implementado el uso de indicadores para medir la calidad ambiental urbana de las ciudades que cuentan con poblaciones mayores a 500.000 habitantes. Estos indicadores se encuentran recopilados en el Índice de Calidad Ambiental Urbana (ICAU), que se ha consolidado como una herramienta significativa para la planificación de las ciudades; además de brindar información sobre el estado de los recursos del ambiente de los que goza cada ciudad evaluada.

Actualmente, el ICAU presenta los resultados únicamente como valores cuantitativos, es decir, no representa la distribución geográfica de cada indicador que lo conforma (entre ellos la superficie de área verde por habitante y el espacio público efectivo), por lo que con esta investigación se busca generar un producto cartográfico que represente la influencia del espacio público y de las superficies verdes en el perímetro urbano de Bogotá. El Observatorio Ambiental de Bogotá ha elaborado mapas que evidencian la manera en la que están distribuidos los indicadores de superficie de área verde por habitante y el espacio público efectivo en la ciudad de Bogotá, pero sus resultados no representan la forma en la que se encuentra distribuida cada variable que conforma estos dos indicadores. Además, estos dos indicadores han sido trabajados por separado aun cuando teóricamente los dos están directamente relacionados.

La importancia de conocer la espacialización de las variables de estos indicadores recae en la obtención de información que determine las zonas críticas en materia de calidad ambiental urbana y la incidencia que estas tienen en la calidad de vida de los ciudadanos.

Es por ello que para el desarrollo de este proyecto se ha planteado en primera instancia un levantamiento de información geográfica del estado actual de los indicadores de superficie de área verde por habitante y el espacio público efectivo, seguido de la verificación y actualización de dicha información. Paso a seguir, se generará el geoproceso en ArcGIS en donde se definirán las variables para la creación de un nuevo indicador en el que se unifiquen los indicadores espacio público efectivo por habitante y superficie de área verde por habitante, y finalmente representar la información sintetizada mediante mapas, que evidencien la distribución del nuevo indicador, siendo posible que las zonas críticas urbanas se manifiesten, entendiendo por zonas críticas las Unidades de Planeamiento Zonal (UPZ) que revelen déficit de espacios de integración y recreación como parques, plazas, plazoletas, escenarios deportivos o elementos de la estructura ecológica principal.

El producto cartográfico obtenido permitirá analizar la influencia del nuevo indicador en la calidad ambiental de la ciudad y en la calidad de vida de los habitantes, y plantear sugerencias encaminadas al ordenamiento territorial, enfatizando la relevancia de estos espacios en el tejido social y ambiental de Bogotá.

\section{Problema de investigación}

Como resultado de la formulación e implementación del ICAU, Colombia cuenta con un grupo de indicadores que sirven para mejorar el conocimiento del estado de las ciudades y dirigir la toma de decisiones que estén orientadas hacia el 
mejoramiento de la calidad ambiental urbana en las mismas. Sin embargo, es evidente que, al contar con gran cantidad de indicadores por determinar, se dificulta la comparación de resultados, lo cual influye en la determinación de la calidad ambiental urbana. Por este motivo, se considera que sería preferible sintetizar algunos de los indicadores que están directamente relacionados como por ejemplo el espacio público efectivo y la superficie de área verde por habitante; ya que estos indicadores hacen referencia al entorno social y al componente paisajístico de las ciudades, elementos que influyen en la calidad de vida de sus habitantes.

En el Informe Nacional de Calidad Ambiental Urbana se evidencia que las autoridades ambientales de cada ciudad manejan los dos indicadores de modo diferente e independiente; es el caso de Bogotá y Cúcuta, cuyas autoridades ambientales suministraron datos de zonas verdes efectivas, que son las áreas blandas del espacio público efectivo, sin contemplar las áreas verdes urbanas privadas; a diferencia del reporte de las ciudades de Medellín, Cali, Barranquilla, Bucaramanga e Ibagué que incluyen en la medición de la superficie verde urbana por habitante no sólo las áreas verdes urbanas públicas sino también las privadas. Esta diferencia tiene influencia en los resultados de la calidad ambiental de cada ciudad dado que los datos reportados no tienen el mismo criterio de consideración de áreas públicas y privadas.

Debido a lo anterior y teniendo en cuenta que dentro del ICAU no se muestra un resultado espacial de la información de los indicadores que lo conforman, ¿son las herramien-

tas SIG adecuadas para sintetizar indicadores relacionados al espacio público como el caso de la superficie de área verde por habitante y espacio público efectivo por habitante para la ciudad de Bogotá D.C?

\section{Objetivos de investigación}

\section{Objetivo general}

Analizar la influencia del espacio público en la calidad ambiental urbana de Bogotá a través del ajuste a los actuales indicadores del ICAU para identificar geográficamente la distribución de las áreas más críticas urbanas.

\section{Objetivos específicos}

- Diseñar un geoproceso en ArcGIS de integración de variables geográficas como propuesta a la síntesis de los actuales indicadores del ICAU en términos de espacio público.

- Identificar los criterios espaciales asociados a los indicadores superficie verde

urbana por habitante y espacio público efectivo por habitante, y su correlación con la calidad ambiental urbana.

- Identificar la distribución del indicador integrado de espacio público para la ciudad de Bogotá, D.C.

\section{Metodología}

El desarrollo de este proyecto se basa en la investigación cuantitativa de tipo exploratoria. La unidad de estudio definida es la ciudad de Bogotá y se hará uso del software ArcGIS. EI diagrama de flujo se presenta en la siguiente figura: 


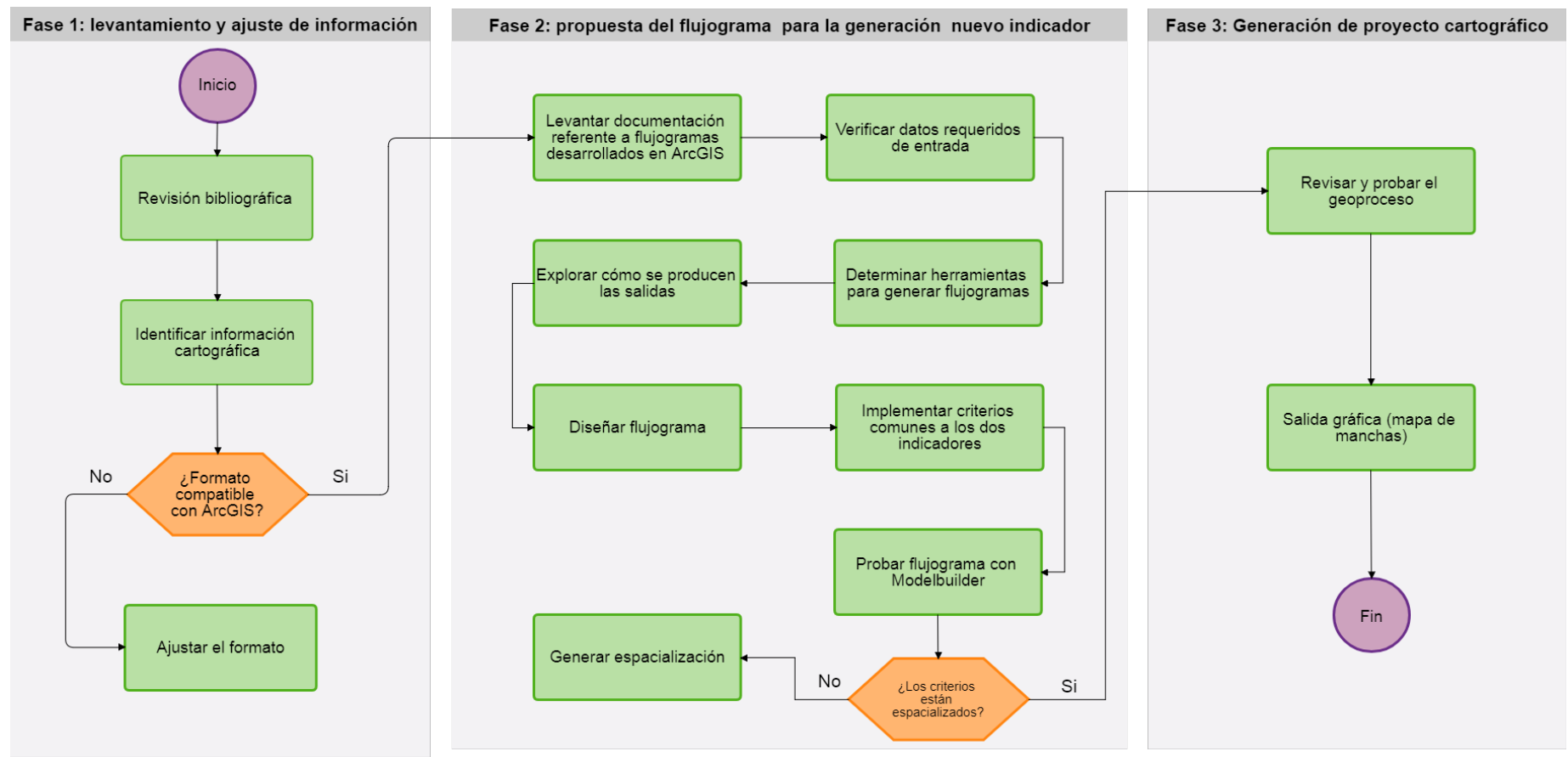

\section{Fase 1: levantamiento y ajuste de información}

En esta primera fase se realizará una revisión bibliográfica de aquellos criterios que hacen parte del indicador de la superficie de área verde por habitante y también del indicador de espacio público efectivo por habitante, pues se asume que estos tienen una fuerte correlación, ya que teóricamente dentro del espacio público por habitante se incluyen los parques y zonas verdes, las cuales a su vez conforman las superficies de área verde de la ciudad.

Por otro lado, se identificará la disponibilidad de información cartográfica y en el caso de que esta información se encuentre en un formato diferente a los que se trabajan en ArcGIS, se procederá a realizar el ajuste de la información a los formatos que se manejan en dicho software.

\section{Fase 2: propuesta del flujograma para la generación nuevo indicador}

En esta segunda fase se realizará el levantamiento de la documentación referente a los diferentes flujogramas desarrollados sobre plataformas ArcGIS para identificar qué datos se requieren en la generación de las entradas de información, cuáles son las herramientas que requiere la generación de estos flujogramas y en qué forma se va a generar la información de salida una vez se trabaje esta por medio de ArcGIS. Para ello es necesario revisar, verificar, probar y analizar los diferentes flujogramas desarrollados en ArcGIS para diseñar un flujograma conveniente en esta plataforma.

Una vez identificados aquellos criterios similares o compartidos y que pueden llegar a integrar un nuevo indicador entre la superficie de área verde por habitante y el espacio público efectivo por habitante se procederá a implementar estos criterios en el flujograma diseñado el cual será revisado y probado en la herramienta de ArcGIS denominada ModelBuilder, con la cual se puede crear y modificar modelos de geoprocesamiento en este software de acuerdo a la definición de procesos e información de entrada y salida. Para ello es necesario definir si las variables o criterios similares entre estos indicadores se encuentran espacializados y en caso de no estarlo, es necesario generar la espacialización de los mismos en ArcGis. 


\section{Fase 3: generación de proyecto cartográfico}

En esta tercera fase se creará el proyecto cartográfico con las variables finales definidas, se revisará y probará el geoproceso para identificar que una vez se corra el proyecto este funcione correctamente; para esto es necesario comprobar que el archivo generado funcione en cualquier dispositivo que contenga la plataforma de ArcGIS y de esta manera obtener finalmente una salida gráfica en la que se muestre el indicador final producto de la sintetización de los dos indicadores trabajados y evaluados.

\section{Principales hallazgos o contribuciones}

- En las localidades en las que hay mayor presencia de parques y zonas verdes su densidad poblacional no es tan alta, por ende, el valor del espacio público efectivo será mayor que en aquellas localidades en las que la densidad poblacional es elevada.

- Los proyectos de revitalización y peatonalización de centros urbanos altamente densificados, toman tiempo y deben ser construidos paso a paso, teniendo siempre como punto de partida un proceso de observación y entendimiento de las dinámicas de la ciudad para poder formular las intervenciones adecuadas de acuerdo con las necesidades específicas.

- Los espacios urbanos de calidad deben ser diseñados para facilitar el uso de cualquier persona y, por lo tanto, seguir criterios que eviten barreras arquitectónicas o espacios inseguros, configurando un entorno físico confortable que fomente el intercambio, la relación social, así como la seguridad e integridad física.

- Es indispensable la constante actualización de la información reportada en los geoportales de las diferentes autoridades, organizaciones e instituciones ambientales, debido a que se evidenció un déficit de información geográfica ambiental, dificultando la medición y confiabilidad de algunos indicadores cuya fuente tiene como registro primario información de cartografía urbana.

- Se recomienda al IDU actualizar la capa de información de plazas y plazoletas de Bogotá, ya que de acuerdo a la clasificación por área que se le da a las plazas y plazoletas, se encuentra que hay plazas que son tomadas como plazoletas y plazoletas que se son trabajadas como plazas.

Bibliografía

Nichol, J (2004). Modeling urban environmental quality in a tropical city. Science Direct. p-1,2,3,6,7. Recuperado de https://www.sciencedirect.com/science/article/pii/S0169204604000957

Decreto 1504. Por el cual se reglamenta el manejo del espacio público en los planes de ordenamiento territorial. Bogotá, Colombia. 4 de agosto de 1998.

Decreto 190. Por el cual se compilan las normas de los Decretos Distritales 619 de 2000 y 469 de 2003, que conforman el Plan de Ordenamiento Territorial de Bogotá, D. C. Bogotá, Colombia. 22 de junio de 2004

Observatorio del Espacio Público de Bogotá. (2017). Reporte Técnico de Indicadores de Espacio Público 2017. Recuperado de http://observatorio.dadep.gov.co/sites/default/files/Reporte-tecnico-2-2017.pdf Consejo Nacional de Política Económica y Social CONPES 3718. Política Nacional de Espacio Público. Bogotá, Colombia. 31 de enero de 2012

Observatorio Ambiental de Bogotá. (2015). Indicadores de Espacio Público en Bogotá. Recuperado de http://oab2.ambientebogota.gov. co/es/documentacion-e-investigaciones/resultado-busqueda/indicadores-de-espacio-publico

Departamento Administrativo de la Defensoría del Espacio Público. (2013). Diagnóstico del espacio público en Bogotá. Recuperado de https://www.dadep.gov.co/publicaciones/diagnostico-del-espacio-pu- 